\title{
A MATRICIAL IDENTITY INVOLVING THE SELF-COMMUTATOR OF A COMMUTING $n$-TUPLE
}

\author{
RAÚL E. CURTO AND RENYI JIAN
}

(Communicated by Eric Bedford)

\begin{abstract}
For a commuting $n$-tuple $a=\left(a_{1}, \ldots, a_{n}\right)$ of elements of a unital $C^{*}$-algebra $\mathscr{A}$, we establish a matricial identity linking the self-commutator of $a$ to the $2^{n-1} \times 2^{n-1}$ matrix $\hat{a}$ that detects the Taylor invertibility of $a$. As a consequence, we obtain a simple proof of a result of D. Xia (Oper. Theory: Adv. Appl. 48 (1990), 423-448), which states that for commuting $t$-hyponormal $n$-tuples, $\sigma_{T}(a)=\sigma_{r}(a)$.
\end{abstract}

Let $a=\left(a_{1}, \ldots, a_{n}\right)$ be a commuting $n$-tuple of elements of a unital $C^{*}$ algebra $\mathscr{A}$. The main result in [Cu1] states that $a$ is invertible on $\mathscr{A}$ (in the sense of Taylor) if and only if a certain $2^{n-1} \times 2^{n-1}$ matrix over $\mathscr{A}$, denoted $\hat{a}$, is invertible. Doubly commuting $n$-tuples (those where $a_{i} a_{j}=a_{j} a_{i}$ and $a_{i} a_{j}^{*}=a_{j}^{*} a_{i}$ for all $i \neq j$ ) admit a much simpler criterion, namely, $a$ is invertible if and only if all $2^{n}$ positive elements $a_{1}^{*} a_{1}+a_{2}^{*} a_{2}+\cdots+a_{n}^{*} a_{n}$, $a_{1}^{*} a_{1}+a_{2}^{*} a_{2}+\cdots+a_{n} a_{n}^{*}, \ldots, a_{1} a_{1}^{*}+a_{2} a_{2}^{*}+\cdots+a_{n}^{*} a_{n}, a_{1} a_{1}^{*}+a_{2} a_{2}^{*}+\cdots+a_{n} a_{n}^{*}$ are invertible. In this note we establish a simple matricial formula linking $\hat{a}$ to the self-commutator of $a$, the $n \times n$ matrix $\left[a^{*}, a\right]:=\left(\left[a_{j}^{*}, a_{i}\right]\right)_{i, j=1}^{n}$, where $\left[a_{j}^{*}, a_{i}\right]:=a_{j}^{*} a_{i}-a_{i} a_{j}^{*}$. As a corollary, we obtain a new proof of a result of D. Xia which states that for $t$-hyponormal $n$-tuples $a$, the Taylor spectrum agrees with the right spectrum.

To explain our results, we need to recall the construction of $\hat{a}$. Rather than repeating the details in [Cu1], we give here an alternate inductive definition. First, for $M$ and $N k \times k$ matrices over $\mathscr{A}$ we let

$$
(M, N)^{\wedge}:=\left(\begin{array}{cc}
M & N \\
-N^{*} & M^{*}
\end{array}\right) .
$$

Now, if $n=1$ we let $\hat{a}:=\left(a_{1}\right)$, and if $n>1$ we let $\hat{a}:=\left(\left(a^{\prime}\right)^{\wedge}, a_{n} I\right)^{\wedge}$, where $a^{\prime}:=\left(a_{1}, \ldots, a_{n-1}\right)$ and $I$ is the $2^{n-2} \times 2^{n-2}$ identity matrix. This definition of $\hat{a}$, slightly different from the one used in [Cu1], is more convenient for our purposes; the following result, nevertheless, still holds.

Received by the editors September 2, 1992.

1991 Mathematics Subject Classification. Primary 47B47, 47A62, 47A10, 47B20, 46L05.

Key words and phrases. Self-commutator, $C^{*}$-algebra, Taylor spectrum, right spectrum, $t$ hyponormal.

The first author's research was partially supported by a grant from NSF.

(C) 1994 American Mathematical Society $0002-9939 / 94 \$ 1.00+\$ .25$ per page 
Lemma 1 [Cu1, Theorem 1]. The commuting $n$-tuple a is invertible on $\mathscr{A}$ if and only if $\hat{a}$ is invertible (as a $2^{n-1} \times 2^{n-1}$ matrix over $\mathscr{A}$ ).

For the next definition we need some notation. If $1 \leq k \leq n$, we let $\mathbf{k}:=$ $\{1, \ldots, k\}, \mathbf{n}:=\{1, \ldots, n\}$, and we let $\operatorname{Inc}(\mathbf{k}, \mathbf{n})$ denote the set of increasing maps from $\mathbf{k}$ to $\mathbf{n}$. We also let $\mathscr{S}_{n}$ denote the set of $n \times n$ diagonal matrices with diagonal entries equal to 1 or -1 .

Definition 2. Let $1 \leq k \leq n$ be given, and let $\varphi \in \operatorname{Inc}(\mathbf{k}, \mathbf{n})$. We define $\varphi^{*}: \mathscr{M}_{n}(\mathscr{A}) \rightarrow \mathscr{M}_{k}(\mathscr{A})$ by $\varphi^{*}(M)_{i, j}:=m_{\varphi(i), \varphi(j)}$, where $M=\left(m_{i j}\right)_{i, j=1}^{n} \in$ $\mathscr{M}_{n}(\mathscr{A})$, and $\varphi_{*}: \mathscr{M}_{k}(\mathscr{A}) \rightarrow \mathscr{M}_{n}(\mathscr{A})$ by

$$
\varphi_{*}(M)_{i, j}:=\left\{\begin{array}{ll}
m_{\varphi^{-1}(i), \varphi^{-1}(j)} & \text { if } i, j \in \varphi(\mathbf{k}) \\
0 & \text { otherwise }
\end{array} \quad\left(M \in \mathscr{M}_{k}(\mathscr{A})\right) .\right.
$$

Definition 3. Let $1 \leq k \leq n$ and let $N \in \mathscr{M}_{k}(\mathscr{A})$ and $M \in \mathscr{M}_{n}(\mathscr{A})$. We say that $N$ is a $k$-generalized principal submatrix of $M$ (abbreviated $k$-GPSM) if there exist $\varphi, \psi \in \operatorname{Inc}(\mathbf{k}, \mathbf{n})$ and a diagonal matrix $D \in \mathscr{S}_{n}$ such that $N=$ $\psi_{*}\left(\varphi^{*}(D M D)\right)$.

Theorem 4. Let $a=\left(a_{1}, \ldots, a_{n}\right)$ be a commuting $n$-tuple of elements of a unital $C^{*}$-algebra $\mathscr{A}$, let $\hat{a}$ be as in Definition 1 , and let $M(a):=\left[a^{*}, a\right]^{t}$. Then

(i) $\hat{a} \hat{a}^{*}=\operatorname{diag}(r)+s$ and

(ii) $\hat{a}^{*} \hat{a}=\operatorname{diag}(r)+t$,

where $r=r_{n}=r(a):=a_{1} a_{1}^{*}+\cdots+a_{n} a_{n}^{*}$, and $s$ and $t$ are sums of GPSM's of $M(a)$.

Before we state the following consequence, we recall that a commuting $n$ tuple $a=\left(a_{1}, \ldots, a_{n}\right)$ is said to be hyponormal if $\left[a^{*}, a\right] \geq 0$ [At, Cu2, CMX, $\mathrm{McCP}$ ] and $t$-hyponormal if $M(a) \geq 0$ [Xia].

Corollary 5 [Xia, Theorem 5]. Assume that $a$ is t-hyponormal. Then $a$ is Taylor invertible on $\mathscr{A}$ if and only if $a_{1} a_{1}^{*}+\cdots+a_{n} a_{n}^{*}$ is invertible.

Proof. Observe that the maps $\varphi^{*}$ and $\psi_{*}$ in Definition 2 preserve positivity, so $s$ and $t$ are both positive. Thus, if $a_{1} a_{1}^{*}+\cdots+a_{n} a_{n}^{*}$ is invertible, we obtain that $\hat{a} \hat{a}^{*}$ and $\hat{a}^{*} \hat{a}$ are invertible, which implies that $a$ is Taylor invertible.

Corollary 6. Let a be a t-hyponormal commuting $n$-tuple of elements of $\mathscr{A}$. Then $\sigma_{r}(a)=\sigma_{T}(a)$.

Proof. Let $\lambda \in \mathbb{C}^{n}$. Observe that $a-\lambda$ is again $t$-hyponormal, so Corollary 5 applies.

\section{Proof OF THEOREM 4}

Our ploy is to use mathematical induction on the number of coordinates. For $n=1$ the result is obvious, as $a_{1}^{*} a_{1}=r+\left[a_{1}^{*}, a_{1}\right]$. Let us consider the case $n=2$. Here

$$
\hat{a} \hat{a}^{*}=\left(\begin{array}{cc}
r & 0 \\
0 & a_{1}^{*} a_{1}+a_{2}^{*} a_{2}
\end{array}\right)=\operatorname{diag}(r)+\left(\begin{array}{cc}
0 & 0 \\
0 & {\left[a_{1}^{*}, a_{1}\right]}
\end{array}\right)+\left(\begin{array}{cc}
0 & 0 \\
0 & {\left[a_{2}^{*}, a_{2}\right]}
\end{array}\right)
$$


and

$$
\hat{a}^{*} \hat{a}=\left(\begin{array}{cc}
a_{1}^{*} a_{1}+a_{2} a_{2}^{*} & {\left[a_{1}^{*}, a_{2}\right]} \\
{\left[a_{2}^{*}, a_{1}\right]} & a_{2}^{*} a_{2}+a_{1} a_{1}^{*}
\end{array}\right)=\operatorname{diag}(r)+M(a),
$$

verifying (i) and (ii). For $n \geq 2$,

$$
\left(a, a_{n+1}\right)^{-}=\left(\begin{array}{cc}
\hat{a} & \operatorname{diag}\left(a_{n+1}\right) \\
-\operatorname{diag}\left(a_{n+1}^{*}\right) & \hat{a}^{*}
\end{array}\right),
$$

so

$$
\begin{aligned}
& \left(a, a_{n+1}\right)^{\mathcal{}}\left[\left(a, a_{n+1}\right)^{\wedge}\right]^{*} \\
& \quad=\left(\begin{array}{cc}
\hat{a} \hat{a}^{*}+\operatorname{diag}\left(a_{n+1} a_{n+1}^{*}\right) & {\left[\operatorname{diag}\left(a_{n+1}\right), \hat{a}\right]} \\
{\left[\hat{a}^{*}, \operatorname{diag}\left(a_{n+1}^{*}\right)\right]} & \hat{a}^{*} \hat{a}+\operatorname{diag}\left(a_{n+1}^{*} a_{n+1}\right)
\end{array}\right)
\end{aligned}
$$

and

$$
\begin{aligned}
& {\left[\left(a, a_{n+1}\right)^{\wedge}\right]^{*}\left(a, a_{n+1}\right)^{\wedge}} \\
& \quad=\left(\begin{array}{cc}
\hat{a}^{*} \hat{a}+\operatorname{diag}\left(a_{n+1} a_{n+1}^{*}\right) & {\left[\hat{a}^{*}, \operatorname{diag}\left(a_{n+1}\right)\right]} \\
{\left[\operatorname{diag}\left(a_{n+1}^{*}\right), \hat{a}\right]} & \hat{a} \hat{a}^{*}+\operatorname{diag}\left(a_{n+1}^{*} a_{n+1}\right)
\end{array}\right) .
\end{aligned}
$$

A moment's thought reveals that a successful inductive argument can be completed once the structure of the commutators $\left[\hat{a}, \operatorname{diag}\left(a_{n+1}\right)\right]$ and $\left[\hat{a}, \operatorname{diag}\left(a_{n+1}^{*}\right)\right]$ is fully determined. (Observe that $r\left(a, a_{n+1}\right)=r(a)+a_{n+1} a_{n+1}^{*}$. ) To reveal this structure, we first need a definition.

Definition 7. Let $1 \leq k \leq n$ and $M \in \mathscr{M}_{n}(\mathscr{A})$. A vector $\mathbf{v} \in \mathscr{A} \otimes \mathbb{C}^{n}$ is said to be a $k$-generalized column vector of $M$ (abbreviated $k$-GCV) if the nonzero entries of $\mathbf{v}$ are part of a column of a $(k+1)$-GPSM of $M$.

Lemma 8. Let $a=\left(a_{1}, \ldots, a_{n}\right)$ be a commuting $n$-tuple in $\mathscr{A}$, and assume that $a_{n+1} \in \mathscr{A}$ commutes with $a_{1}, \ldots, a_{n}$. Then

(i) If $n$ is odd, $\left[\operatorname{diag}\left(a_{n+1}\right), \hat{a}\right]$ consists of $\left(\begin{array}{c}n \\ n-1\end{array}\right)(n-1)-G C V ' s,\left(\begin{array}{c}n \\ n-3\end{array}\right)(n-$ 3)-GCV's, ..., ( $\left(\begin{array}{l}n \\ 2\end{array}\right)$ 2-GCV's of $M\left(a, a_{n+1}\right)$, and a zero column vector (the last column $)$, and $\left[\hat{a}^{*}, \operatorname{diag}\left(a_{n+1}\right)\right]$ consists of $\left(\begin{array}{l}n \\ n\end{array}\right) \quad n-G C V ' s,\left(\begin{array}{c}n \\ n-2\end{array}\right)(n-2)-$ $G C V^{\prime} s, \ldots,\left(\begin{array}{l}n \\ 1\end{array}\right) \quad 1-G C V$ 's of $M\left(a, a_{n+1}\right)$.

(ii) If $n$ is even, $\left[\operatorname{diag}\left(a_{n+1}\right), \hat{a}\right]$ consists of $\left(\begin{array}{c}n \\ n-1\end{array}\right) \quad(n-1)-G C V ' s, \quad\left(\begin{array}{c}n \\ n-3\end{array}\right)$ $(n-3)-G C V^{\prime} s, \ldots,\left(\begin{array}{l}n \\ 1\end{array}\right)$ 1-GCV's of $M\left(a, a_{n+1}\right)$, and $\left[\hat{a}^{*}, \operatorname{diag}\left(a_{n+1}\right)\right]$ consists of $\left(\begin{array}{l}n \\ n\end{array}\right) \quad n-G C V$ 's, $\left(\begin{array}{c}n \\ n-2\end{array}\right)(n-2)-G C V$ 's, ..., ( $\left.\begin{array}{l}n \\ 2\end{array}\right)$ 2-GCV's of $M\left(a, a_{n+1}\right)$, and a zero column vector (the last column).

Proof. We use mathematical induction. The conclusions certainly hold for $n=$ 2 and $n=3$; assume first that $n$ is odd, and that the assertions are true for all $k<n$. From the inductive definition of $\hat{a}$, it follows at once that

$$
\left[\operatorname{diag}\left(a_{n+1}\right), \hat{a}\right]=\left(\begin{array}{cc}
{\left[\operatorname{diag}\left(a_{n+1}\right),\left(a^{\prime}\right)^{\wedge}\right]} & 0 \\
\operatorname{diag}\left(\left[a_{n}^{*}, a_{n+1}\right]\right) & {\left[\operatorname{diag}\left(a_{n+1}\right),\left[\left(a^{\prime}\right)^{\wedge}\right]^{*}\right]}
\end{array}\right) .
$$

By the inductive hypothesis, the first $2^{n-2}$ columns of $\left[\operatorname{diag}\left(a_{n+1}\right), \hat{a}\right]$ contain $\left(\begin{array}{l}n-1 \\ n-2\end{array}\right) \quad(n-1)$-GCV's, ..., (c $\left.\begin{array}{c}n-1 \\ 2\end{array}\right)$ 3-GCV's, and one 1-GCV of $M\left(a, a_{n+1}\right)$. Similarly, the last $2^{n-2}$ columns of $\left[\operatorname{diag}\left(a_{n+1}\right), \hat{a}\right]$ contain $\left(\begin{array}{c}n-1 \\ n-1\end{array}\right)(n-1)-$ GCV's, ..., $\left(\begin{array}{c}n-1 \\ 1\end{array}\right)$ 1-GCV's of $M\left(a, a_{n+1}\right)$. The conclusion then follows by recalling that $\left(\begin{array}{c}n-1 \\ k-1\end{array}\right)+\left(\begin{array}{c}n-1 \\ k\end{array}\right)=\left(\begin{array}{l}n \\ k\end{array}\right)$. The cases of $n$ odd, and the two cases corresponding to $\left[\hat{a}^{*}, \operatorname{diag}\left(a_{n+1}\right)\right]$ are entirely similar. 
We can now conclude the proof of Theorem 4. Assume that $n$ is even. By inductive hypothesis and formula (2), we see that

$$
\begin{aligned}
& {\left[\left(a, a_{n+1}\right)^{\wedge}\right]^{*}\left(a, a_{n+1}\right)^{\wedge}} \\
& \quad=\operatorname{diag}\left(r_{n+1}\right)+\left(\begin{array}{cc}
t_{n} & {\left[\hat{a}^{*}, \operatorname{diag}\left(a_{n+1}\right)\right]} \\
{\left[\operatorname{diag}\left(a_{n+1}^{*}\right), \hat{a}\right]} & s_{n}+\operatorname{diag}\left(\left[a_{n+1}^{*}, a_{n+1}\right]\right)
\end{array}\right) .
\end{aligned}
$$

Now observe that each GPSM of $M\left(a, a_{n+1}\right)$ is either a GPSM of $M(a)$ or it involves $a_{n+1}$ in both a column and a column. Since $t_{n}$ as well as $s_{n}$ are sums of GPSM's of $M(a)$, an application of Lemma 8 shows that

$$
\left(\begin{array}{cc}
t_{n} & {\left[\hat{a}^{*}, \operatorname{diag}\left(a_{n+1}\right)\right]} \\
{\left[\operatorname{diag}\left(a_{n+1}^{*}\right), \hat{a}\right]} & s_{n}+\operatorname{diag}\left(\left[a_{n+1}^{*}, a_{n+1}\right]\right)
\end{array}\right)
$$

can also be written as a sum of GPSM's of $M\left(a, a_{n+1}\right)$. Thus,

$$
\left[\left(a, a_{n+1}\right)^{\wedge}\right]^{*}\left(a, a_{n+1}\right)^{\wedge}-\operatorname{diag}\left(r_{n+1}\right)
$$

is a sum of GPSM's of $M\left(a, a_{n+1}\right)$. A similar argument holds in the remaining cases.

Remark 9. Formulas (1) and (2), and the proofs of Theorem 4 and Lemma 8 show that $s_{n}$ and $t_{n}$ can be written recursively in terms of $a_{1}, \ldots, a_{n}$ starting from $\left[\left(a_{1}, a_{2}\right)^{\wedge}\right]^{*}\left[\left(a_{1}, a_{2}\right)^{\wedge}\right]$ and $\left.\left[\left(a_{1}, a_{2}\right)^{\wedge}\right]\left[a_{1}, a_{2}\right)^{\wedge}\right]^{*}$.

\section{ACKNOWLEDGMENT}

This work was done while the second author was on sabbatical leave at the University of Iowa. He wishes to express his appreciation to the colleagues in the Department of Mathematics for their hospitality during the 1991-92 academic year.

\section{REFERENCES}

[At] A. Athavale, On joint hyponormality of operators, Proc. Amer. Math. Soc. 103 (1988), 417-423.

[Cu1] R. Curto, Spectral permanence for joint spectra, Trans. Amer. Math. Soc. 270 (1982), 659-665.

[Cu2] __ Joint hyponormality: a bridge between hyponormality and subnormality, Proc. Sympos. Pure Math., vol. 51, part 2, Amer. Math. Soc., Providence, RI, 1990, pp. 69-91.

[CMX] R. Curto, P. Muhly, and J. Xia, Hyponormal pairs of commuting operators, Oper. Theory: Adv. Appl., vol. 35, Birkhäuser Verlag, Basel, 1988, pp. 1-22.

[McCP] S. McCullough and V. Paulsen, A note on joint hyponormality, Proc. Amer. Math. Soc. 107 (1989), 187-195.

[Xia] D. Xia, On some classes of hyponormal tuples of commuting operators, Oper. Theory: Adv. Appl., vol. 48, Birkhäuser Verlag, Basel, 1990, pp. 423-448.

Department of Mathematics, University of Iowa, Iowa City, Iowa 52242

E-mail address: curto@math.uiowa.edu

Department of Mathematics, Guizhou Normal University, Guiyang 550001, People's REPUBLIC OF CHINA 